\title{
Reconstruction of Neural Circuits Using Serial Block-Face Scanning Electron Microscopy
}

${ }^{\dagger}$ These authors contributed equally to this work.

*Correspondence to:

Lee KJ,

Tel: +82-53-980-8320

Fax: +82-53-980-8309

E-mail: relaylee@kbri.re.kr

Received June 21, 2016

Revised June 25, 2016

Accepted June 25, 2016

\author{
Gyu Hyun $\mathrm{Kim}^{\dagger}$, Sang-Hoon Lee ${ }^{1, \dagger}$, Kea Joo Lee* \\ Laboratory of Synaptic Circuit Plasticity, Department of Structure \& Function of Neural Network, \\ Korea Brain Research Institute, Daegu 41068, Korea \\ ${ }^{1}$ Research Equipment Core Facility Team, Korea Brain Research Institute, Daegu 41068, Korea
}

\begin{abstract}
Electron microscopy is currently the only available technique with a spatial resolution sufficient to identify fine neuronal processes and synaptic structures in densely packed neuropil. For large-scale volume reconstruction of neuronal connectivity, serial blockface scanning electron microscopy allows us to acquire thousands of serial images in an automated fashion and reconstruct neural circuits faster by reducing the alignment task. Here we introduce the whole reconstruction procedure of synaptic network in the rat hippocampal CA1 area and discuss technical issues to be resolved for improving image quality and segmentation. Compared to the serial section transmission electron microscopy, serial block-face scanning electron microscopy produced much reliable threedimensional data sets and accelerated reconstruction by reducing the need of alignment and distortion adjustment. This approach will generate invaluable information on organizational features of our connectomes as well as diverse neurological disorders caused by synaptic impairments.
\end{abstract}

Key Words: Electron microscopy, Hippocampus, Synapse, Connectomics, Mapping

\section{INTRODUCTION}

Since Ramón y Cajal proposed the concept that neurons in the brain communicate with each other through intercellular junctions called synapses more than a century ago, synaptic structures could finally be visualized with the advent of transmission electron microscopy (TEM) (Blackstad, 1965). Thereafter, TEM of brain samples has been an essential approach for observing fine synaptic structures including presynaptic vesicles, post-synaptic density, synaptic cleft, mitochondria, smooth endoplasmic reticulum, spine apparatus, and polyribosomes (Frotscher et al., 2014; Steward \& Levy, 1982). In addition, by means of the electron microscopy (EM)'s superior resolving power to identify and trace individual axons and dendritic processes in densely packed neuropil, substantial attempts has been made to reconstruct neuronal circuitry in both physiological and neuropathological conditions (Kim et al., 2014; Lee et al., 2005a, 2005b, 2013). However, large-scale EM volume reconstruction has been challenged by the daunting task of acquiring thousands of serial sections and manually tracing the stack of aligned images.

Recent technological developments have significantly improved the quality of large-scale EM data and reduced the time-consuming processes such as manually aligning image stacks. These advanced EM techniques including serial section TEM with a Camera Array (TEMCA), automated tapecollecting ultramicrotome (ATUM), serial block-face scanning EM (SB-SEM) and focused ion beam scanning EM (FIB-SEM) (Briggman \& Bock, 2012) has been employed to produce

This research was supported by KBRI basic research program funded by the Ministry of Science, ICT \& Future Planning (No. 2231-415), the Korea Health Technology R\&D Project funded by the Ministry of Health and Welfare (HI14C1135), Basic Science Research Program funded by the Ministry of Education (2014R1A1A2058740), and the R\&D Convergence Program of National Research Council of Science \& Technology of Republic of Korea.

(a) This is an open-access article distributed under the terms of the Creative Commons Attribution Non-Commercial License (http://creativecommons.org/licenses/by-nc/4.0) which permits unrestricted noncommercial use, distribution, and reproduction in any medium, provided the original work is properly cited.

Copyrights $(2016$ by Korean Society of Microscopy 
unprecedentedly large volume EM data sets of nervous tissues (Cardona et al., 2010; Denk \& Horstmann, 2004; Hayworth et al., 2014; Knott et al., 2008). While each method has specialized advantages and inherent limits, SB-SEM recently installed at Korea Brain Research Institute promises to make EM reconstruction much faster and easier. Instead of cutting and handling with serial sections the outside of EM, an ultramicrotome mounted inside the vacuum chamber of SBSEM enables to cut ultrathin sections repeatedly (typically 30 50 nm-thick) from the face of the resin-embedded tissue block. Between being sectioned, the surface of the sample block is imaged by detection of back-scattered electrons. This approach allows us to acquire thousands of serial EM images in an automated fashion, because it dramatically reduces the need for the alignment and ordering stages of serial section TEM. Although the lateral resolution of SB-SEM is slightly lower than TEM, it provides a sufficient resolving power to trace synaptic profiles and small-caliber axons (Cardona et al., 2010; Denk \& Horstmann, 2004; Hayworth et al., 2014; Knott et al., 2008).

Combined with other functional assays including live imaging and electrophysiology, reconstructions of neuronal wiring diagrams using SB-SEM might reveal how the brain integrates multiple sensory stimuli, stores memory, and controls behavior. Furthermore, such mappings of wiring diagrams could identify the location of neuronal circuits impaired in neurological disorders such as Alzheimer's disease and autism. Therefore, large volume EM is an indispensable technology to understand what the precise distributions and weights of individual synapses are on dendritic segments, what neurons are connected together, which neurons are involved in a particular neural processing, and how those neurons are organized into functional networks.

In this report, we introduce how we reconstructed rat hippocampal neural circuits using SB-SEM and raise some technical issues to be resolved for future experiments.

\section{MATERIALS AND METHODS}

Sample preparation methods in SB-SEM are similar to that in TEM with some modifications to enhance signal for backscattered electron imaging of epoxy embedded brain
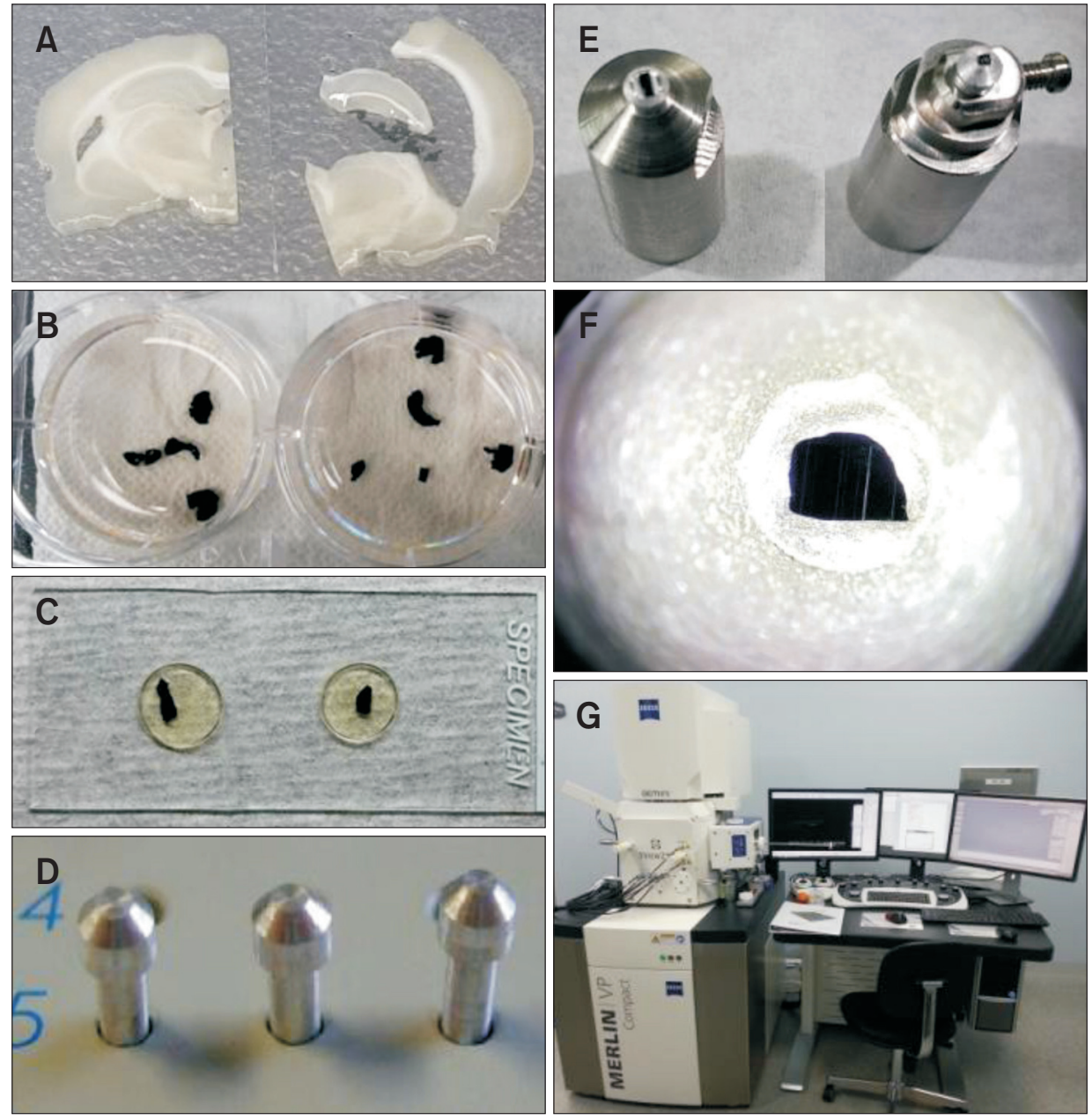

Fig. 1. Sample preparation for serial block-face scanning electron microscopy (SB-SEM). (A) Dissection of hippocampal tissue. (B) Tissues blackened after post fixing with osmium. (C) Flat-embedded hippocampal tissues. (D, E) Hippocampal tissues mounted on the sample rivets for SB-SEM. (F) Surface-trimmed specimen grounded with silver paint followed by a gold-palladium sputter coating. (G) SBSEM installed at Korea Brain Research Institute. 
tissue at low accelerating voltage. An intensive osmium treatment and en bloc heavy metal staining are required for volume imaging under SEM to avoid severe charging and achieve the high membrane contrast (Wilke et al., 2013). Three-month-old Sprague Dawley male rats $(n=2)$ were deeply anesthetized and intracardially perfused with $2 \%$ paraformaldehyde and $2.5 \%$ glutaraldehyde in $0.15 \mathrm{M}$ cacodylate buffer ( $\mathrm{pH} 7.4)$. Brain slices (100 150 $\mu$ m-thick) were made with a vibratome in ice-cold $0.15 \mathrm{M}$ cacodylate buffer, and small pieces of hippocampal tissues were stored overnight in the same fixative at $4^{\circ} \mathrm{C}$. After being washed, samples were placed in cacodylate buffer containing $2 \%$ OsO4/1.5\% potassium ferrocyanide for 1 hour. Tissues were placed in $1 \%$ thiocarbohydrazide (TCH) (Ted Pella, USA) solution for 20 minutes and then placed in $2 \%$ aqueous OsO4 for 30 minutes. Thereafter, tissues were incubated in $1 \%$ uranyl acetate at overnight and lead aspartate solution at for 30 minutes to enhance membrane contrast as described previously (Walton, 1979). Hippocampal tissues were dehydrated using a graded series of ethanol (50\%, 70\%, $95 \%$, and $100 \%$ ), infiltrated with acetone, mixture of resin and acetone, and $100 \%$ resin. The resin was prepared from the Epon 812 kit (EMS, USA) following the manufacturer's instructions. Samples are then mounted between liquid release agent-coated glass slides and embedded in a thin layer of fresh resin and cured at $60^{\circ} \mathrm{C}$ for 2 days. Small pieces of resin embedded tissues are mounted on aluminum rivet (Gatan, USA) using cyanoacrylate glue. For observations with SB-SEM and data analyses, the lower surface of sample was grounded with silver paint and gold-palladium sputtering to increase conductivity, and imaged in field emission-SEM (Merlin; Carl Zeiss, Germany) equipped with 3View2 system (Gatan). The serial EM images obtained were aligned with Image J software with Fiji plugins (http:// fiji.sc/wiki/index.php/Fiji), and segmentation and image analyses were performed with reconstruct program that is freely available (http://synapses.clm.utexas.edu/software-0). Animal experiments were performed in accordance with the guidelines for animal research set out in the Korean Academy of Medical Sciences, and approved by the ethics committee of the Korean Brain Research Institute (IACUC-15-00003).

\section{RESULTS}

SB-SEM detects backscattered electron signal from the surface of the plastic resin-embedded tissue block at low accelerating voltage (typically $1 \sim 3 \mathrm{kV}$ ) (Fig. 1). Therefore, the sample should fulfill the requirements for the enhancement of membrane contrast and reduction of surface charging and beam damage effects. A combination of osmium, TCH, heavy metal staining, silver paste grounding, and sputter coating (10 nm layer of gold) greatly enhanced the image quality and membrane contrast of serial EM images (Fig. 2). The electrical charging and beam damage effects for imaging nonconducting samples could also be minimized by adjusting imaging parameters for SEM such as accelerating voltage, section thickness, magnification, and pixel time. As expected, SB-SEM images we obtained at the initial trials exhibited adequate resolution to observe presynaptic vesicles, postsynaptic densities, synaptic cleft, mitochondria, and spine apparatus (Fig. 2). To reconstruct local synaptic connectivity, we collected 500 serial EM images at the accelerating voltage of $2.0 \mathrm{kV}$, covering $38.5 \times 38.5 \times 25.0 \mu \mathrm{m}$ volume in CA1 area stratum radiatum of the rat hippocampus (approximately 37,000 $\mu^{3}$ ). Trials to image the samples at accelerating voltages of $<1.7 \mathrm{kV}$ exhibited excessive noise in this condition. The size of each image was 5,000×5,000 pixels (z-step size: $50 \mathrm{~nm}$, pixel resolution: $7.7 \mathrm{~nm}$, pixel dwell time: $1 \mu \mathrm{s}$, magnification: 7,000×, lens aperture: 30 $\mu \mathrm{m})$. Before acquiring each image stack, we confirmed the volume location based on CA1 pyramidal cell layer at lower magnification $(\sim 500 \times)$. The serial EM images were converted to Tiff format, automatically aligned to correct the marginal image drift and distortion using Image J software with Fiji plugins (http://fiji.sc/wiki/index.php/Fiji), and then cropped

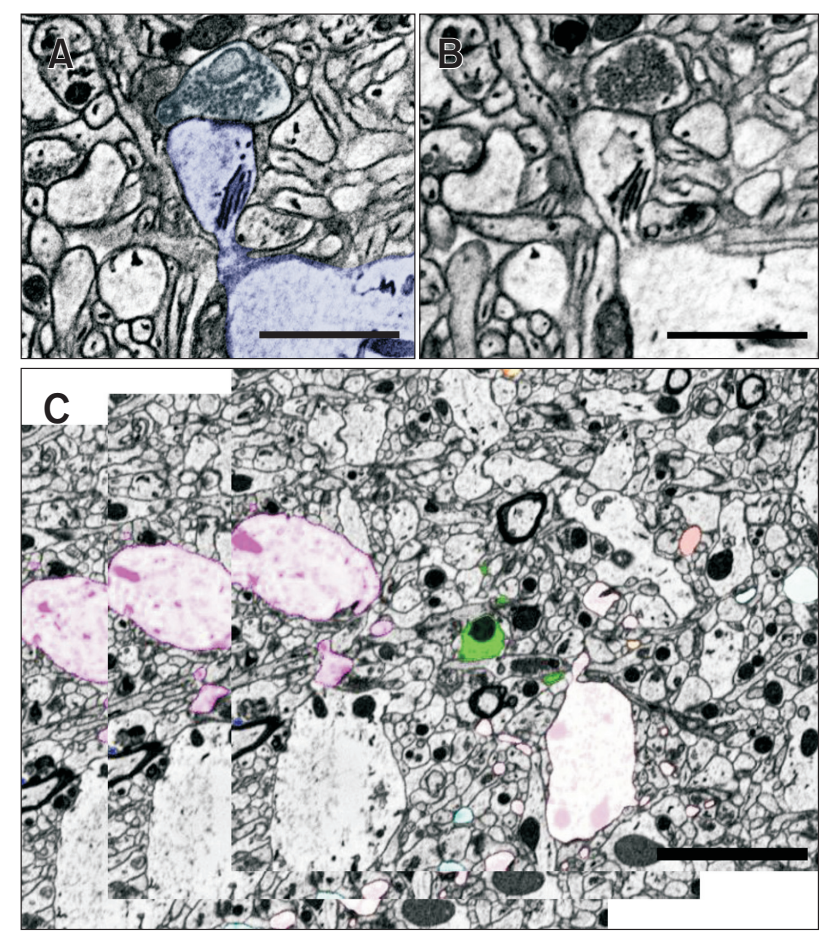

Fig. 2. Serial electron microscopy (EM) images and tracing of CA1 dendritic profiles. (A, B) Adjacent (100 nm apart) EM images clearly showing an axonal bouton containing presynaptic vesicles, postsynaptic density, and dendritic spines with spine apparatus. Note the enhanced membrane contrast following osmium-thiocarbohydrazide-osmium method. (C) Serial tracing of dendritic profiles of pyramidal neurons in CA1 area. Scale bars $=0.5 \mu \mathrm{m}(\mathrm{A}, \mathrm{B})$ and $5 \mu \mathrm{m}(\mathrm{C})$. 

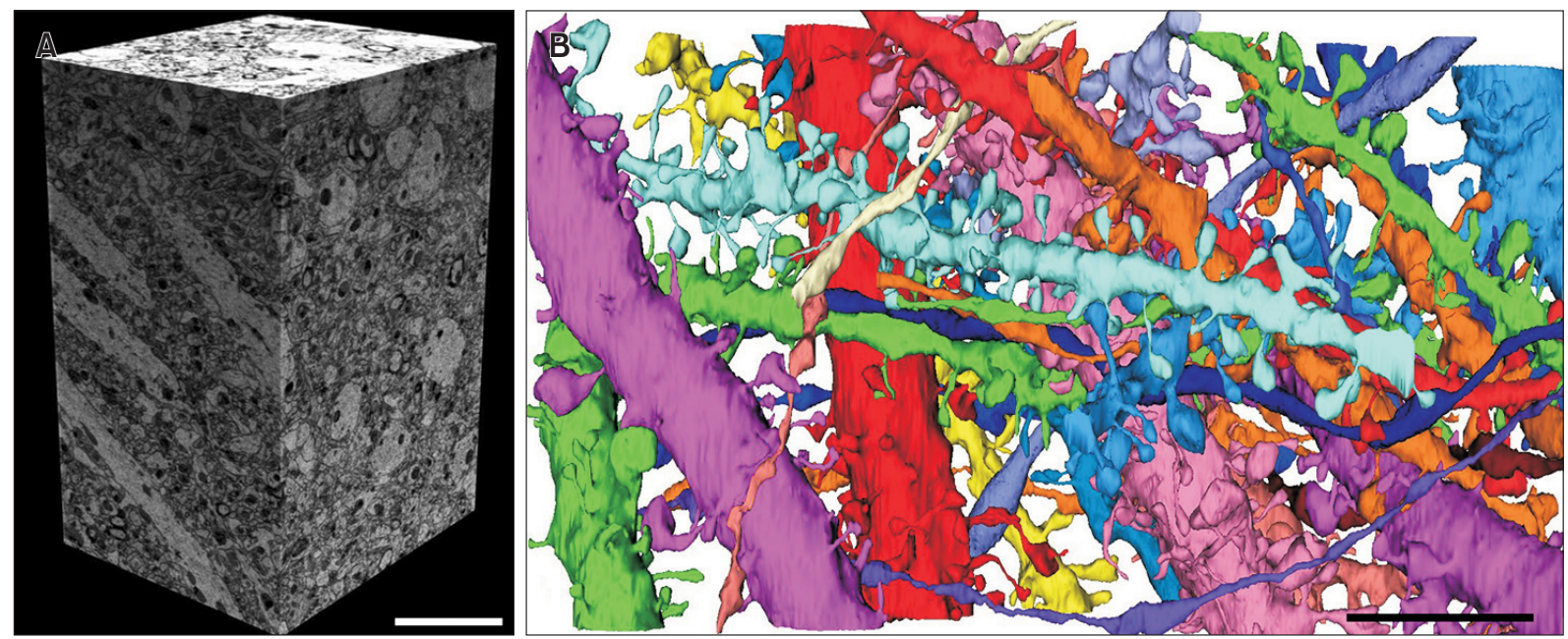

Fig. 3. Reconstruction of local synaptic network. (A) Serial block-face scanning electron microscopy images of rat hippocampus arranged into a $17.4 \times 13.1 \times 25.0 \mu \mathrm{m}$ cuboid $\left(\sim 5,700 \mu \mathrm{m}^{3}\right)$. Scale bar=5 $\mu \mathrm{m}$. (B) Manual reconstruction of local synaptic circuits in hippocampal CAl area. Scale bar=5 $\mu \mathrm{m}$.

into a small volume $(17.4 \times 13.1 \times 25.0 \mu \mathrm{m}$ volume; $\sim 5,700$ $\left.\mu \mathrm{m}^{3}\right)$. Then, we manually traced pre- and post-synaptic profiles in serial images using Reconstruct program (http:// synapses.clm.utexas.edu/software-0). The morphology of dendritic segments and synapses formed between presynaptic axon terminals and dendritic spines were clearly observed and precisely reconstructed (Fig. 3). The final aligned volume and sparse reconstruction of local hippocampal synaptic circuits is available online (https://www.youtube.com/ watch? $=2$ SOymAdZdrs).

\section{DISCUSSION}

In the present study, we were able to obtain three-dimensional volume data set $\left(\sim 37,000 \mu \mathrm{m}^{3}\right)$ in the rat hippocampal CA1 area using SB-SEM in an automated fashion, and successfully reconstructed local synaptic network with sufficient resolution to identify sub-synaptic structures and reliably trace fine neuronal processes. Therefore, this approach is a useful option for large-scale structural mapping of neuronal connectivity as the field of image could further enlarged by tiled imaging and stitching partially-overlapped adjacent images together. One caveat in SB-SEM is that non-conductive plastic resinembedded samples are prone to experiencing electrical charging and beam damage that negatively affect the lateral resolution of EM images. This issue may be mitigated by a recent discovery of conductive resin that not only reduces the electrical charging, but also enhances the resolution of images obtained for volume imaging with SEM (Nguyen et al., 2016). Although SB-SEM significantly reduces the time-consuming alignment step, the major bottleneck of synaptic circuit reconstruction has been a manual tracing of serial image stacks that is a formidable task in middle sized labs. Currently, a number of researchers are developing an automated EM segmentation algorithm for dense reconstruction of large tissue volume (Jones et al., 2015; Mishchenko, 2011). The integration of artificial intelligence based fully-automated image segmentation algorithms to the large SB-SEM datasets would achieve unprecedentedly large volume reconstruction of mammalian neural circuits. Moreover, the recent advent of SEM with 61 parallel electron beams would further revolutionize the image acquisition speed and realize to image sample in the centimeter scale at nanometer resolution $(\sim 4$ $\mathrm{nm} / \mathrm{pixel}$ ) (Marx, 2013).

In conclusion, we have successfully reconstructed synaptic circuits using SB-SEM. This approach will be used to understand the principles of brain functions and identify impaired synaptic connections in neurodegenerative disorders.

\section{CONFLICT OF INTEREST}

No potential conflict of interest relevant to this article was reported.

\section{ACKNOWLEDGMENTS}

The authors thank Shin KM, Bae BK, Kim CW, Lee CH, Kim YK and Nam YJ for their segmentation and visualization of neural processes. EM data were acquired in the Advanced Neural Imaging Center at KBRI. 


\section{REFERENCES}

Blackstad T W (1965) Electron microscopy and biological structure research. Tidsskr. Nor. Laegeforen. 85, 97-103.

Briggman K L and Bock D D (2012) Volume electron microscopy for neuronal circuit reconstruction. Curr. Opin. Neurobiol. 22, 154-161.

Cardona A, Saalfeld S, Preibisch S, Schmid B, Cheng A, Pulokas J, Tomancak P, and Hartenstein V (2010) An integrated micro- and macroarchitectural analysis of the Drosophila brain by computerassisted serial section electron microscopy. PLoS Biol. 8. doi: 10.1371/journal.pbio.1000502.

Denk W and Horstmann H (2004) Serial block-face scanning electron microscopy to reconstruct three-dimensional tissue nanostructure. PLoS Biol. 2, e329.

Frotscher M, Studer D, Graber W, Chai X, Nestel S, and Zhao S (2014) Fine structure of synapses on dendritic spines. Front. Neuroanat. 8 , 94.

Hayworth K J, Morgan J L, Schalek R, Berger D R, Hildebrand D G, and Lichtman J W (2014) Imaging ATUM ultrathin section libraries with WaferMapper: a multi-scale approach to EM reconstruction of neural circuits. Front. Neural Circuits 8, 68.

Jones C, Liu T, Cohan N W, Ellisman M, and Tasdizen T (2015) Efficient semi-automatic 3D segmentation for neuron tracing in electron microscopy images. J. Neurosci. Methods 246, 13-21.

Kim H W, Kim D S, and Rhyu I J (2014) Electron tomography and synapse study. Appl. Microsc. 44, 83-87.

Knott G, Marchman H, Wall D, and Lich B (2008) Serial section scanning electron microscopy of adult brain tissue using focused ion beam milling. J. Neurosci. 28, 2959-2964.

Lee K J, Kweon H S, Kang J S, and Rhyu I J (2005b) 3-Dimensional reconstruction of parallel fiber-purkinje cell synapses using highvoltage electron microscopy. Korean J. Electron. Microsc. 35, 31-39.

Lee K J, Park C H, and Rhyu I J (2005a) Efficient three-dimensional reconstruction of synapse with high-voltage electron microscopy. J. Electron Microsc. 54, 139-141.

Lee K J, Park I S, Kim H, Greenough W T, Pak D T, and Rhyu I J (2013) Motor skill training induces coordinated strengthening and weakening between neighboring synapses. J. Neurosci. 33, 9794 9799.

Marx V (2013) Neurobiology: brain mapping in high resolution. Nature 503, 147-152.

Mishchenko Y (2011) Reconstruction of complete connectivity matrix for connectomics by sampling neural connectivity with fluorescent synaptic markers. J. Neurosci. Methods 196, 289-302.

Nguyen H B, Thai T Q, Saitoh S, Wu B, Saitoh Y, Shimo S, Fujitani H, Otobe H, and Ohno N (2016) Conductive resins improve charging and resolution of acquired images in electron microscopic volume imaging. Sci. Rep. 6, 23721

Steward O and Levy W B (1982) Preferential localization of polyribosomes under the base of dendritic spines in granule cells of the dentate gyrus. J. Neurosci. 2, 284-291.

Walton J (1979) Lead aspartate, an en bloc contrast stain particularly useful for ultrastructural enzymology. J. Histochem. Cytochem. 27, 1337-1342.

Wilke S A, Antonios J K, Bushong E A, Badkoobehi A, Malek E, Hwang M, Terada M, Ellisman M H, and Ghosh A (2013) Deconstructing complexity: serial block-face electron microscopic analysis of the hippocampal mossy fiber synapse. J. Neurosci. 33, 507-522. 